LIAMES 6 - pp. 77-99, Primavera 2006

Félix Quesada Castillo

(Univ. Nac. Mayor de San Marcos, Lima - Perî)

\title{
Desarrollo del futuro en las lenguas de los Andes Centrales: del significado lexical al significado gramatical
}

\begin{abstract}
This article explains the development of future paradigms in a group of non-genetically related Amerindian languages of the Central Andes: Quechua, Aymara, Jaqaru, Uru-Chipaya, Mochica, and Puquina. We argue that the diachronic development of the future tense is similar in all of these languages: it is a grammaticalization process which consists in the generalization or universalization of a particular lexical meaning. Our investigation has arrived at the following results: a) The morphemes of future time in the Central Andean languages are the result of a process of semantic and phonetic change (fusion and reduction) of a limited set of lexical elements (roots); b) The meanings of the lexical forms involved in the development of the future refer to movement, desire, intention, necessity, supposition, command, and possession; c) The nuclear meaning of the future is that of prediction, which results from a process of semantic extension of the lexical meanings; d) The nuclear meaning is accompanied by various modal meanings (desire, intention, obligation, necessity, and supposition) that are retentions of the meanings of the lexical sources; e) In some languages such as Mochica, the forms with future meaning still exhibit their condition as roots or particles, which is further evidence in support of our basic claim.
\end{abstract}

KEYWORDS: Andean languages; Future tense; Grammaticalization; Semantic Change.

RESUMEN: Elucidamos el desarrollo de los paradigmas del futuro en un grupo de lenguas amerindias de los Andes centrales no relacionadas genéticamente: quechua, aimara, jaqaru, uru-chipaya, mochica y puquina. El desarrollo del futuro en las lenguas estudiadas consiste en el cambio del significado lexical al significado gramatical, fenómeno universal que se denomina gramaticalización. En consecuencia, el proceso de formulación del futuro es similar en las lenguas y la gramaticalización consiste en un proceso de generalización o universalización de un significado lexical particular. Nuestra investigación ha llegado a los siguientes resultados: a) Los morfemas del tiempo futuro en las lenguas de los Andes Centrales constituyen el resultado del proceso del cambio semántico y fonético (fusión y reducción) a partir de un conjunto limitado de elementos lexicales (raíces); b) El significado de las formas léxicas implicadas en el desarrollo del futuro se refieren a movimiento, deseo, intención, obligación, necesidad, suposición, comando y posesión; c) El significado nuclear del futuro es el de la predicción que resulta de un proceso de extensión semántica de los significados lexicales; d) El significado nuclear está acompañado de varios significados modales (deseo, intención, obligación, necesidad y suposición) que son retenciones de los significados de las fuentes lexicales; e) En algunas lenguas como el mochica, las formas con significado de futuro aún exhiben su condición de raíces o partículas, evidencia que apoya nuestra hipótesis.

PALABRAS-CLAVE: Lenguas Andinas; Tiempo futuro; Gramaticalización; Cambio semántico. 


\section{INTRODUCCIÓN}

Las lenguas de los Andes Centrales incluidas en este estudio son el quechua, el mochica y jaqaru; el puquina y el aimara, de la zona meridional. El puquina parece ser la única lengua que tuvo su origen y su difusión en el área meridional, mientras que se postula que el área original del aimara estuvo en los Andes del Perú Central. Estas lenguas, como resultado de un contacto secular o por relación genética entre algunas de ellas, exhiben rasgos comunes. Actualmente, el mochica y el puquina son lenguas extintas, mientras que el quechua, el aimara y el jaqaru son lenguas habladas que muestran diferentes magnitudes demográficas.

Al presente, no existe ningún estudio específico que dé cuenta del origen y el proceso de formación del tiempo futuro en estas lenguas. Los escasos enfoques históricos proponen la reconstrucción de los proto-morfemas (Parker, 1969) o establecen las variantes en el actual aimara (Briggs, 1976, 1993 y Cerrón-Palomino, 1993).

El presente estudio tiene por objetivo identificar y describir las formas verbales del futuro, vigentes en las lenguas habladas del área estudiada y su registro en las fuentes escritas existentes sobre las lenguas extintas más arriba mencionadas. A partir de las formas actuales y de las registradas en los testimonios escritos, se postula la fuente y el proceso de formación del futuro hasta alcanzar las formas actuales.

Con el fin de alcanzar el objetivo de describir y explicar la formación del tiempo futuro, se procederá a desarrollar las siguientes tareas: (i) establecer la metodología del estudio, (ii) determinar la hipótesis de la investigación y (iii) postular los principios formales y semánticos que gobiernan el desarrollo del futuro.

\section{METODOLOGÍAYDATOS}

\subsection{Procedimiento metodológico}

El objetivo del estudio exige un enfoque interlingüístico e interdialectal. El enfoque interlingüístico consiste en el estudio de la categoría temporal de futuro a través de las lenguas que conforman el conjunto de las lenguas de los Andes Centrales y dos lenguas del área meridional. En esta etapa de la investigación no se han incluido al cholón, lengua de los Andes Centrales, y el uru-chipaya, lengua hablada en la zona meridional de los Andes. Para la caracterización del futuro en cada lengua no extinta, fue necesario el estudio de este tiempo a través de los dialectos. La aplicación de este procedimiento ha sido sólo posible en las lenguas actualmente del área: quechua, aimara y jaqaru.

El enfoque interdialectal ha permitido dar cuenta del desarrollo del futuro en cada lengua y el interlingüístico en el conjunto del área.

La apelación al procedimiento interlingüístico responde a la necesidad de establecer, como resultado de la comparación de lenguas no emparentadas, los procesos y principios universales en la formación del morfema del futuro. Dentro del marco de este enfoque y con el fin de elucidar el origen y desarrollo del tiempo futuro, hemos apelado a los principios del cambio lingüístico y a los procedimientos de reconstrucción diacrónica. 
Consecuentemente, el enfoque interlingüístico ha conducido a la comparación de lenguas no relacionadas genéticamente como una extensión del método comparativo tradicional.

Ahora bien, para los estudios interdialectales dentro del quechua y el aimara se ha utilizado el método comparativo tradicional. En este sentido, se procede tanto con la comparación lingüística ortodoxa y con la implementada por los estudios de los universales.

\subsection{Datos}

En esta sección efectuamos una breve presentación sobre la naturaleza de los datos requeridos y el procedimiento seguido en la recolección de los mismos.

Los datos que han constituido el objeto de nuestro análisis y la base de nuestra generalización están conformados por el corpus recolectado de los hablantes a través de los paradigmas y los textos grabados y transcritos. El otro tipo de información está constituida por las gramáticas léxicas y textos existentes tanto para las lenguas habladas como para las extintas.

Aparte de la información valiosa y necesaria que se dispone sobre las lenguas habladas (quechua, aimara y jaqaru) existe una limitación en la información sobre el mochica y el puquina, actualmente lenguas extintas. Sobre el mochica se cuenta con gramáticas y textos (Carrera, 1939 y Middendorf, 1892), mientras que para el puquina existe un esbozo gramatical (Grasserie, 1894) muy fragmentario y un conjunto de textos religiosos. Estos materiales, especialmente en el caso del puquina, son muy valiosos, pero insuficientes, para contar con una información que nos permita dar cuenta del desarrollo de esta forma verbal satisfactoriamente.

\section{EL FUTURO EN LAS LENGUAS DE LOS ANDES CENTRALES}

En esta parte del trabajo proponemos una descripción de las formas verbales correspondientes al tiempo futuro en las lenguas del área denominada Andes Centrales, y dos lenguas de la región Collao sobre la base de la información recolectada.

Las lenguas de los Andes Centrales estudiadas no presentan un marcador flexional regular del futuro, sino que los paradigmas exhiben una configuración heteróclita y, en el caso del mochica, el futuro se forma añadiendo partículas al verbo, es decir, son aún construcciones perifrásticas. Esta característica nos revela la etapa relativamente reciente del futuro.

El puquina parece disponer de un morfema para marcar la flexión del futuro, pero los datos fragmentarios de que disponemos no nos permiten proponer una caracterización de este tiempo.

Las lenguas quechua, el aimara-jaqaru, mochica y puquina conforman el área lingüística que hemos denominado Andes Centrales, una sección del área andina (Büttner, 1983). Aunque estas lenguas están relacionadas arealmente por haber compartido una extensa zona del área andina, sin embargo no existe una vinculación histórica confirmada.

El aimara y el jaqaru, a pesar de su gran cercanía, en virtud de su relación genética, conforman la familia aru (Cerrón-Palomino, 1982, Torero, 1970 y Hardman, 1966) o aimara 
(Cerrón-Palomino, 1993). La similaridad entre el quechua y el aimara-jaqaru, especialmente en el léxico, y la presencia de las series aspiradas y glotalizadas obstruyentes no continuas ha motivado la hipótesis de la relación genética entre ambas lenguas o familias lingüísticas; sin embargo, esta hipótesis se debilita por el prolongado contacto entre ellas, hecho que puede haber motivado el desarrollo de las similaridades aludidas.

Por tanto, la caracterización de la formación del futuro mediante la comparación de las cuatro lenguas no emparentadas genéticamente exhibe rasgos tipológicos y universales.

De las cinco lenguas (en este caso, considerando el aimara y el jaqaru como dos lenguas hermanas) comparadas, cuatro (80\%) indican el futuro mediante morfemas ligados, o flexión sufijante. Es decir, usan el futuro morfológico, según la terminología de algunos autores. Entre las cinco lenguas, el mochica marca el futuro mediante morfemas no ligados: las partículas ca y chäm, cuyo significado es 'tener'. Sin embargo, al lado del futuro morfológico se ha registrado el futuro perifrástico en el quechua, el jaqaru y el aimara.

En la mayoría de las lenguas analizadas, la flexión del futuro es heterogénea, por cuanto existe una marca específica para cada persona, como es el caso del quechua y las lenguas de la familia aru.

El estudio del futuro a través de las lenguas de las áreas establecidas nos lleva a postular que la flexión del futuro es el resultado de procesos fonéticos y semánticos consistentes en la mutación del significado lexical de la unidad lingüística al significado gramatical de predicción.

\subsection{El futuro en el quechua}

Con el fin de dar cuenta del desarrollo del futuro en las lenguas estudiadas, hemos conducido un estudio interdialectal del fenómeno. En el Cuadro 1(cf. Apéndice) se presentan muestras representativas de las variaciones dialectales de los marcadores del futuro en el quechua. Las variedades del grupo NORTE, CENTRO y SUR tratan de representar las variaciones en cada una de la tres zonas geográficas en términos generales. Sin embargo, debemos advertir que ellas no expresan todas las diferencias que se pueden registrar, dado que éstas constituyen un continuum.

Del examen de las formas utilizadas en la expresión del significado de futuro, se concluye que el quechua expresa dicho significado por medio de las formas que se muestran a continuación: 
Quesada: Desarrollo del futuro en las lenguas de los Andes Centrales

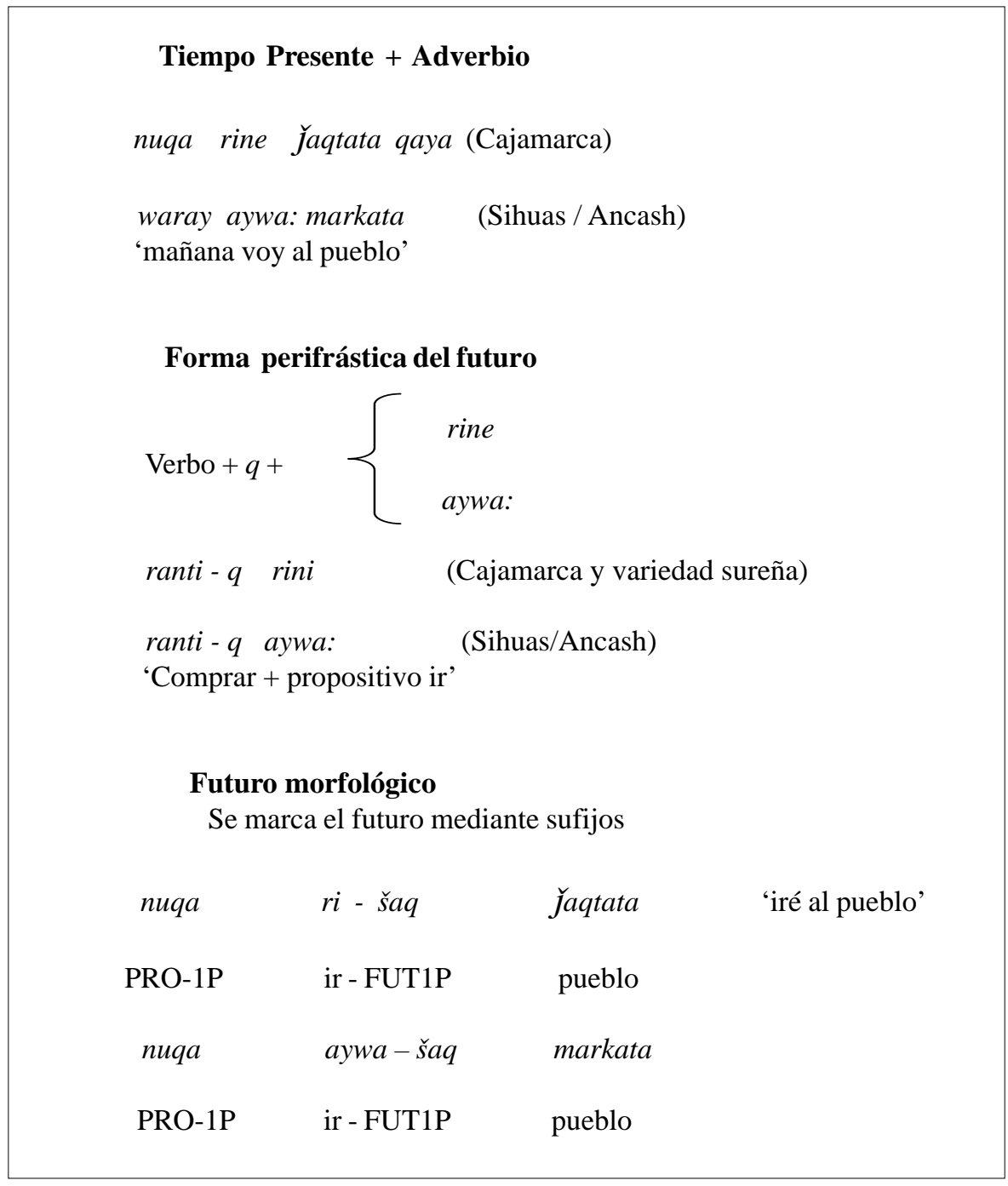

Sobre la base del análisis sincrónico y diacrónico de los paradigmas del futuro morfológico de los dialectos quechuas que están representados por la muestra en el Cuadro 1(cf. Apéndice), postulamos que el futuro en esta lengua está constituido por un sistema flexivo de tres morfemas: -̌̌aq (primera persona 1p), -nqa (tercera persona $3 p$ ) y-̌̌un (inclusivo (incl.)).

Como se puede observar en la muestra del Cuadro 1, éste constituiría el sistema más anterior del cual se han derivado las formas en los dialectos quechuas actuales, como lo muestran las variedades del cajamarquino y el dialecto de Sihuas. 
Las descripciones previas incluyen en el paradigma del futuro también a la segunda persona ( $-n k i)$, y se sostiene que la marca de 2 p de este tiempo es el mismo del presente. Con la inclusión de $2 \mathrm{p}$, el paradigma que se ha establecido está constituido por cuatro miembros, más los plurales de 2 p y 3 p, como se exhibe en el siguiente paradigma (Parker, 1976):

$\begin{array}{ll}1 \mathrm{p} & -\check{s} a q \\ 2 \mathrm{p} & -n k i \\ 3 \mathrm{p} & -n q a \\ \text { Incl. } & -\check{s} u n \\ \text { Excl. } & -y a: \check{s} a q \\ 2 \mathrm{pp} & - \text { yanki } \\ 3 \mathrm{pp} & - \text { yanqa }\end{array}$

Del análisis del paradigma del futuro en el quechua, podemos establecer las siguientes conclusiones:

1.- Los paradigmas del futuro en las diferentes variedades del quechua presentan ligeras diferencias. Estas diferencias pueden explicarse, básicamente, mediante procesos fonológicos. La mayor diferencia semántica (además de la fonológica con su posterior morfologización) se da entre las variedades ecuatoriana/chachapoyana y las otras variedades de la muestra. En el primer grupo se ha eliminado la diferencia entre el inclusivo y exclusivo, que sí perdura en las demás variedades de la muestra.

2.- El paradigma del futuro en el quechua es caracterizado como un sistema defectivo y, siguiendo a Bybee y Pagliuca (1985b), se le considera un paradigma "heterogéneo". Es un sistema defectivo porque no todos los miembros del paradigma tienen marca de futuro (en particular, la 2p). Asimismo, es un sistema heterogéneo en virtud de que presenta marcadores específico para cada persona.

3.- Existe en el quechua actual dos formas para expresar el significado del tiempo futuro: a) El futuro morfológico que está constituido por un paradigma flexivo de 3 miembros y b) el futuro perifrástico constituido por la construcción verbo- $q+r i$ - como en tanta-ta rantiq rini (Cajamarca) "voy a comprar pan”. Además del significado de "predicción”, esta forma perifrástica retiene su significado original de "movimiento". Es decir, implica futuridad, pero mantiene el significado de "propósito" o "movimiento con un determinado propósito".

\subsection{El futuro en el aimara}

La flexión de futuro es expresada por un marcador que presenta alternancias de acuerdo con la flexión de persona y la diversidad dialectal. Los datos disponibles sobre el fenómeno nos conducen a postular que los marcadores de la flexión del futuro marcan persona y tiempo (posterior al momento del enunciado). Excepto la segunda persona (2p) y presumiblemente la tercera (3p) existe un solo marcador para tiempo y persona correspondiente, como se puede observar en el siguiente ejemplo correspondiente al aimara de la zona de La Paz (Padres de Maryknoll et al 1969): 


$\begin{array}{ll}1 \mathrm{p} & \text { sara }-:-w a^{l} \\ 2 \mathrm{p} & \text { sara }-:-t w a \\ 3 \mathrm{p} & \text { sara-ni-wa } \\ \text { Incl (4p) } & \text { sara-ñani }\end{array}$

En este paradigma, el futuro de $1 \mathrm{p}$ y $2 \mathrm{p}$ es - : - (en otras variedades el futuro de $2 \mathrm{p}$ se marca con $-n)$; de 3p, $-n i$; y del inclusivo, $-\tilde{n} a n i$. Como se observa en los paradigmas, -ta es la marca de segunda persona como en los otros tiempos. Por ahora, se podría postular que $-i$ es la marca de 3p y $-n$, del futuro.

En la descripción del futuro en el aimara se observa que esta lengua revela una gran variabilidad en la marcación de la flexión de este tiempo (cf. Cuadro 2 del Apéndice). La mayor variabilidad se da en las personas $1 \mathrm{p}$ y $2 \mathrm{p}$. A continuación presentamos las alternancias más importantes:

$$
\begin{aligned}
& 1 \mathrm{p}-:-\sim-j a \sim-x a \sim-y a \sim-n h a \sim \tilde{n} a \\
& 2 \mathrm{p}-:-\sim-j a \sim-j a-:-n \sim n h a
\end{aligned}
$$

El marcador de $3 \mathrm{p}-n i$ es universal, pues es la única marca para todas las variedades aimaras. La forma del inclusivo varía entre - ñani y -tana, aunque el primer morfo es el más generalizado.

El problema en la descripción de la 1p y 2p está en determinar si las alternancias dialectales derivan de una forma única más anterior o son indicadoras de desarrollos paralelos en la formación del futuro. Si las alternancias fuesen predecibles mediante procesos fonéticos, sería posible postular una fuente única en la formación del futuro en el aimara. De otro modo deberá postularse que las diferencias regionales sugieren diferentes fuentes y procesos en la formación de la flexión del futuro. Este problema será tratado en la sección 4. Desde el punto semántico, además del significado básico de una "acción a realizarse en el porvenir" (probabilidad y predicción), los hablantes tienden a traducir los enunciados que contienen formas del futuro mediante la construcción: ir $+a+$ infinitivo, como se ve a continuación.

$$
\begin{array}{ll}
\text { sara-ni-wa } & \text { "él va a ir" } \\
\text { sara- :-wa } & \text { "voy a ir" }
\end{array}
$$

\subsection{El futuro en el jaqaru}

El tiempo futuro en esta lengua está constituido por un paradigma de sufijos, en el cual el rasgo nasal es la única característica común: -nha [-na] (1p), -mata (2p), -ni (3p) y -tana (incl. o 4p). (cf. Cuadro 3, Apéndice).

La $1 p(-n h a)$ es común a algunas variedades del aimara. La segunda persona podría segmentarse en ma- (marca de futuro) y -ta (marca de 2p). Se propone esta segmentación como resultado de la comparación con la segunda persona del presente:

1 : Indica alargamiento vocálico, en el texto aveces aparece precedido de guión - :, en otras precedido y seguido del guión - : - para indicar que se está identificando um morfema. 
/-ta/ e imperativo $-m a(2 \mathrm{p} \Longrightarrow 3 \mathrm{p})$.

La 3p se marca con /-ni/ como en el aimara. Este sufijo puede considerarse como el marcador de $3 p$ y tiempo futuro a la vez o puede ser analizado en $n$ - (marca de futuro) y el sufijo $-i$ como marcador de $3 \mathrm{p}$. El inclusivo (4p) es idéntico al marcador de la misma persona del presente. De la observación del paradigma, se podría suponer que la $(4 \longrightarrow 3 \mathrm{p})$ no se marca para futuro y se podría concluir que cada miembro está constituido por sufijo diferente y específico para cada persona: cada sufijo del paradigma marca persona y tiempo futuro, con la posibilidad de excluir $2 \mathrm{p}$ y $3 \mathrm{p}$ que pueden segmentarse para tiempo y persona, aunque ésta es una simple sugerencia por ahora.

De acuerdo con Hardman (1982), estudiosa del jaqaru que ha ofrecido una interesante y excelente descripción de esta lengua, el tiempo futuro se refiere a un futuro simple y "expresa una obligación suave". El significado de obligatoriedad constituye un indicador de la posible fuente de la formación de la flexión del futuro en esta lengua (cf. Cuadro 3).

\subsection{El futuro en el mochica y en el puquina}

Para analizar esta lengua, contamos con la gramática de Carrera (1939) y de Middendorf (1892). Ambos autores coinciden en gran parte en la caracterización del paradigma de futuro. Carrera considera el futuro del verbo substantivo ( $e$ y sus variantes) que se forma mediante la adición de la partícula $c a(k a)$. (cf. Cuadro 4, Apéndice).

$\begin{array}{lllll}1 \mathrm{p} & \text { moiñ } & e & c a & \text { 'yo seré' } \\ 2 \mathrm{p} & \text { tzhang } & e & c a & \text { 'tú serás' } \\ 3 \mathrm{p} & \text { aiof } & & c a & \text { 'aquel será' } \\ 1 \mathrm{ppl} & \text { maich } & \text { ang } & c a & \text { 'nosotros seremos' } \\ 2 \mathrm{ppl} & \text { tzhaich } & e z \text { chi } & c a & \text { 'vosotros seréis' } \\ 3 \mathrm{ppl} & \text { aiong } & \text { an e } & c a & \text { 'aquellos serán' }\end{array}$

La segunda manera de formar el futuro es mediante la adición de la partícula chäm al presente. El significado de la referida partícula es 'tener'; es decir, está relacionado con la idea de obligatoriedad.

De acuerdo con Middendorf y Carrera existe otra forma para el futuro: con otro verbo substantivo (copulativo) y los verbos no copulativos. La primera forma consistirá en la adición de la partícula $\mathrm{ca}(\mathrm{ka})$ a la forma denominada presente (Middendorf, 1892).

La tercera forma del futuro (denominado por Middendorf primer futuro) se estructura antecediendo a la raíz verbal las desinencias, a las cuales se antepone t:

\begin{tabular}{|c|c|c|c|}
\hline $1 \mathrm{p}$ & $\underline{t i n}$ & met & 'yo traeré' \\
\hline $2 p$ & $\underline{t} a z$ & met & ‘tú traerás’ \\
\hline $3 p$ & $\underline{t}$ tang & met & 'él traerá' \\
\hline $1 \mathrm{ppl}$ & $\underline{t i x}$ & met & 'nosotros traeremos' \\
\hline $2 \mathrm{ppl}$ & $\underline{t} a z$ & metchi & 'vosotros traeréis' \\
\hline $3 \mathrm{ppl}$ & $\underline{t}$ ang & metan & 'aquellos traerán' \\
\hline
\end{tabular}


La información sobre el futuro en el puquina la ofrece Grasserie (1894). Los datos sobre el futuro se reducen a las referencias que ofrece el autor en la sección de la gramática del puquina. De acuerdo con la información de Grasserie, el futuro está indicado por que, c, gue:

$$
\begin{array}{ll}
\text { cuya-gue-p-anch } & \text { 'tú amarás' } \\
\text { confessa-gue-p-anch } & \text { 'tú confesarás' } \\
\text { confes-sasca-gue-anch } & \text { 'me confesará' }
\end{array}
$$

No podemos ofrecer mayor información sobre su ocurrencia y las alternancias. De acuerdo con la traducción, su valor semántico equivale al futuro morfológico o sintáctico en otras lenguas como el quechua y el castellano.

\section{FORMACIÓN DEL FUTURO EN LAS LENGUAS DE LOS ANDES CENTRALES: BASESLEXICALES}

En esta sección nos concentramos en la reconstrucción de la forma más antigua del futuro morfológico, es decir, de la flexión del tiempo futuro en el quechua y en la lengua aimara o aru. Luego de la reconstrucción del proto-sistema que propongamos, avanzaremos hasta las fuentes o bases de la formación del futuro en estas lenguas.

\subsection{Reconstrucción del proto-sistema del futuro en el quechua}

En el quechua es posible reconstruir la etapa del proto-quechua y a partir de este estado podemos proponer uno anterior al proto-quechua, que hemos denominado pre-proto-quechua (Quesada, 1988), que constituiría la forma más antigua.

Del examen interdialectal del paradigma del futuro se observa variación en el nivel fonológico (véase el Cuadro 1, Apéndice), se asume que esta variabilidad es el resultado de los cambios fonológicos operados en el quechua como parte de su desarrollo. Respecto del aspecto semántico, el significado básico está presente a través de los dialectos, pero existe una variación leve en algunos rasgos semánticos, especialmente en el significado modal.

El establecimiento de las correspondencias en los paradigmas del futuro en los dialectos que exhiben las mayores diferencias ha conducido a postular determinados procesos fonéticos y éstos han hecho posible la reconstrucción del paradigma del proto-quechua.

Los procesos que nos permiten la reconstrucción de las proto-formas del paradigma son:

1 Sonorización de las oclusivas sordas $\underline{\mathbf{k}}$ y $\mathbf{q}$ en los dialectos del quechua del Norte y Oriente: Cajamarca-Cañaris, San Martín, Chachapoyas y Ecuador.

2 Reducción de $\underline{\mathbf{k}}$, consistente en el proceso de espirantización. La velar oclusiva $\underline{\mathbf{k}}$ se convierte en $\underline{\mathbf{s}}$. Este proceso ocurre en los miembros del paradigma, que exhiben el segmento velar oclusivo. Este fenómeno ocurre en el quechua sureño (Cusco, Bolivia y Santiago del Estero) y San Martín. 
3 Debilitamiento y caída de la uvular q en algunos dialectos del quechua de Ancash.

4 Despalatalización de š en el quechua sureño que origina la presencia de $\underline{\mathbf{s}}$ en lugar de s: $\check{s}>$ s.

La postulación de estos procesos ocurridos en los paradigmas del futuro de los diferentes dialectos, conduce a la reconstrucción del paradigma del proto-quechua:

$$
\begin{array}{lc}
1 \mathrm{p} & * \text {-šaq } \\
2 \mathrm{p} & \emptyset \\
3 \mathrm{p} & *-\text { nqa } \\
\text { incl } & * \text {-šun }
\end{array}
$$

Este paradigma coincide con los paradigmas de algunas variedades conservadoras que han retenido los rasgos del paradigma del proto-quechua.

Dado que todos los dialectos comparten el paradigma reconstruido, se asume que al momento de la ramificación del quechua en los dialectos actuales ya se había formado el paradigma del futuro con los rasgos que exhibe el sistema reconstruido.

\subsubsection{El pre-proto-quechua: las fuentes léxicas del futuro}

Establecido el paradigma del proto-quechua, surge la cuestión: ¿Cuáles son las formas más antiguas a partir de las cuales se ha desarrollado la flexión del futuro del quechua? Postulamos que el paradigma del futuro en esta lengua es el resultado del desarrollo de elementos lexicales y construcciones del pre-proto-quechua. Estos procesos son de carácter semántico y fonético. Esta hipótesis se basa en la existencia de formas análogas en los dialectos quechuas actuales cuyos significados guardan cierta relación con los significados del futuro. El segundo fundamento está constituido por los estudios de los universales en el desarrollo del significado gramatical (Bybee y Pagliuca, 1985a, 1985b).

Con base en estos fundamentos, postulamos que la primera persona y el inclusivo derivan de los elementos lexicales: ša - 'movimiento', más el propositivo - q, y el inclusivo, de una partícula (posiblemente verbal) šun. La tercera persona es la construcción de -n, más la partícula qa con un significado específico (obligación, deseo o sugerencia). Esta partícula puede estar relacionada con un significado contextual de qa topicalizador o la partícula vinculada a la forma que se añadía a š, que al presente ha perdido su significado original, pero ha formado el prefecto š-qa. Sin embargo, semánticamente, sería más plausible que la forma original de n sería na por su significado obligativo y de futuridad.

La teoría postula que el futuro y el significado gramatical tienen como fuentes verbos que significan deseo, movimiento, posesión y obligación (Bybee y Pagliuca, 1985b). Sobre esta base y la coexistencia del futuro con los morfemas que conservan su significado y el futuro viene a ser la forma derivada mediante el cambio semántico y fonético, proponemos las siguientes fuentes léxicas del paradigma flexional del futuro en el quechua: 
La fuente del futuro de primera persona (1p) es el verbo de movimiento ša- y el propositivo -q. La raíz ša- que indica movimiento, actualmente se usa con este significado original en construcciones como

$$
\begin{array}{ll}
\text { Šarikuy } & \text { 'levántate' } \\
\text { šamuynari } & \text { 'ven ya pues' }
\end{array}
$$

Es decir en los tiempos históricos (pre-proto-quechua) era una clase de verbo de movimiento. Esta raíz se combinaba con -q que con ciertos verbos transitivos significa 'propositivo de acción' y por tanto implica la realización de la acción posterior al enunciado o constituye un tipo de predicción del evento.

Actualmente al lado del futuro morfológico, ocurre el futuro sintáctico o perifrástico. Esta construcción esta formado por un verbo de movimiento y un verbo transitivo modificado por el propositivo -q.

$$
\begin{array}{ll}
\text { rantiq } & \text { rine } \\
\text { aywa: } & \text { rantikuq }
\end{array}
$$

Es decir que en la formación del futuro se requiere un verbo de movimiento y la presencia del propositivo - $\mathrm{q}$.

En el marco de esta estructura morfosintáctica y semántica, la expresión del futuro en el pre-proto-quechua se realizaba mediante una construcción en la que intervenía un verbo de movimiento al cual se añadía el propositivo -q, forma que se posponía a un verbo:

$$
\text { Verbo + ša- 'movimiento' }+q
$$

La construcción ša $+q$ derivó en el significado de futuro.

En los dialectos actuales se da el futuro perifrástico cuyos componentes son: un verbo +q más el verbo de movimiento ri- o aywa- . Estos componentes debieron estar presentes en una expresión de futuro en el pre-proto-quechua.

Es decir la predicción se expresaba mediante una construcción mikušaq ( 'comeré' o 'voy a comer'). Posteriormente la forma libre šaq, mediante un proceso de fusión de šaq con el verbo, se convirtió en un sufijo marcador de la $1 \mathrm{p}$ de futuro. Esto nos lleva a concluir que la construcción $\mathrm{V}+$ šaq sufre un proceso de fusión fonética que se correlaciona con el proceso de formación de šaq en un morfema flexivo de futuro, proceso que implica un cambio de significado lexical en el significado gramatical, es decir de morfema de futuro.

Los dialectos quechuas modernos han desarrollado el futuro perifrástico con significado de modalidad diferente al del futuro morfológico. Esta forma del futuro consiste en el verbo de movimiento ir (aywa- o ri-) flexionado para persona, más otro verbo al cual se pospone el propositivo $-\mathrm{q}$ :

$$
\text { rantiq rine 'voy a ir' }
$$


En esta forma ha reemplazado ri- o aywa- a ša- del pre-proto-quechua, el que al haberse gramaticalizado y fusionado con el propositivo -q funciona en ciertos dialectos modernos con el valor de propositivo que se añade al verbo:

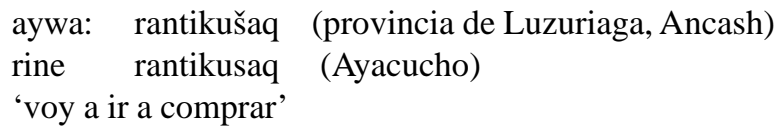

Para el marcador de futuro de $3 p$-nqa se propone la forma no marcada para tiempo mikun + qa que en este caso sería el uso de qa con el significado de 'deseo', 'sugestión' u 'obligación'. Esta hipótesis se apoya en el hecho que esta construcción retiene el significado de obligación o sugerencia. Veamos el caso en el quechua de Cajamarca:

$\begin{array}{ll}\text { mikunqa } & \text { 'que coma' } \\ \text { puñunqa } & \text { 'que duerma' } \\ \text { rinqa } & \text { 'que vaya' } \\ \text { sarikunqa } & \text { 'que se levante' }\end{array}$

Estos ejemplos constituirían la evidencia de que estas construcciones originalmente significaban 'sugerencia' y 'obligación'. A partir de estos significados se desarrollo el significado de futuro, es decir como resultado del cambio semántico. Si los significados de obligación y futuridad no están vinculados a qa, sería razonable postular que $\mathbf{n}$ se deriva del nominalizador -na por elisión de la vocal.

De otro lado, el inclusivo tiene como fuente la expresión šun que significa incitación, sugerencia, obligación. Es decir tiene un significado cercano al modal shall del inglés.

Luego por un proceso similar a los anteriores se convirtió en el marcador del futuro: fusión fonética entre la raíz y la partícula šun, en correlación con el cambio semántico de significado léxico al significado gramatical.

\subsection{Formación del futuro en el aimara}

Seleccionando al dialecto de Huancané al azar tenemos el siguiente paradigma del futuro.

$\begin{array}{ll}1 \mathrm{p} & -: \\ 2 \mathrm{p} & -: \text { ta } \sim \text {-nta } \\ 3 \mathrm{p} . & - \text { ni } \\ 4 \mathrm{p}(\text { incl) } & - \text { ñani }\end{array}$

Este paradigma nos conduce a formular la siguiente hipótesis:

1.- Sobre la base de la alternancia -ja (velar) -xa (post velar) se podría postular que estos son reflejos de una secuencia obstruyente + vocal. Sin embargo, no hemos podido identificar hasta ahora el tal segmento obstruyente y por tanto esta hipótesis quedaría descartada. 
2.- De otro lado, la alternancia -: -ja -xa -nha (velar nasal) además de la presencia de -n en la segunda persona llevaría a postular que la primera persona tiene como fuente la primera persona del posesivo -na. Esta afirmación tiene sustento por cuanto una de las fuentes es el elemento léxico con significado de posesión. Sin embargo no podríamos dar cuenta de la 3p -ni y el inclusivo -ñani, dado que no se podría justificar el cambio $n>\tilde{n}$ delante de vocal posterior. Por esta razón y en vista de otras evidencias abandonamos esta hipótesis y proponemos la hipótesis alternativa que exponemos en 3.

3.- En razón de que en la variedad denominada salinas se alterna -: con -ña y en Morocomarca con -ya, es decir un elemento palatal, postulamos que la flexión de futuro en el aimara tiene dos fuentes: el posesor -ni y el infinitivo -ña que uno de sus significados es de obligación y potencial. En la 1p, 2p y el inclusivo se habría formado añadiendo al verbo el infinitivo y posteriormente se produce la fusión fonética y la despalatalización en la $1 \mathrm{p}$ y $2 p$ y el desarrollo del significado gramatical de futuro. La presencia de -ni en la $4 p$ (inclusivo) simplemente indica la combinación de la marca de 1p y la $3 p$ que es un posible camino en la formación del inclusivo.

Esta hipótesis es apoyada por los datos ofrecidos en Huayhua (1991), quien en su condición de nativo hablante del aimara sostiene que actualmente en Conima (Provincia de Huancané), el futuro presenta alternancias que pueden constituir evidencia de que el marcador del futuro tiene su origen en el infinitivo.

Para $1 \mathrm{p}, 2 \mathrm{p}$ y $4 \mathrm{p}$ o inclusivo se tiene:

$$
\begin{aligned}
& \text { 1p apa-na-tha } \sim \text { apa }-\mathrm{n}-\mathrm{ja} \sim \text { apa-: } \\
& 2 \mathrm{p} \text { apa-na-ta } \sim \text { apa - } \mathrm{n}-\mathrm{ta} \sim \text { apa }: \text {-ta } \\
& \text { 3p /apa-n-i/ apa - n-i -na -ni } \\
& \text { incl. apa-ñani } \sim \text { apa-na-ni }
\end{aligned}
$$

A base de todo lo expuesto, podemos postular que las protoformas de donde han derivado los morfemas del futuro son:

$$
\begin{array}{ll}
*_{- \text {ña }} & \text { infinitivo } \\
*_{\text {-ni }} & \text { poseer }
\end{array}
$$

\subsection{Formación del futuro en el jaqaru}

La comparación del paradigma del jaqaru con el del aimara nos conduce a establecer una similaridad en el futuro en ambas lenguas; sin embargo, respecto al jaqaru se podría postular que las personas $1 \mathrm{p}$ y $2 \mathrm{p}$ se derivan del obligativo -nha ( $\mathrm{n}$ ) y -ma respectivamente, ya que el léxico con significado de obligación es una de las fuentes en la formación del futuro.

El inclusivo consiste en la presencia de la forma imperativo o de la combinación de -ta $2 p$ del imperativo y el obligativo -na de primera persona antes de su velarización. 
La tercera persona tendría la misma fuente y el mismo desarrollo. Es decir provendría del posesor -ni que habría desarrollado su significado de futuro. Nuestra hipótesis se apoya en el hecho que las formas de futuro en esta lengua posee significado de obligación que constituiría un componente semántico del futuro como un fenómeno de retención del significado original de obligación, en cambio el significado de predicción (del futuro) constituiría una extensión, es decir el cambio semántico en el desarrollo del futuro.

\subsection{Formación del futuro en el mochica}

El futuro en el mochica constituye un caso diferente respecto a las otras lenguas objeto de este estudio. En el quechua, aimara, jaqaru y el puquina el morfema de futuro está indicado por sufijos, es decir son morfemas ligados. En el mochica del siglo XVII, el futuro se indicaba mediante partículas o formas libres. Es decir no constituía un fenómeno estrictamente morfológico sino que se manifestaba mediante una construcción sintáctica.

Tal como hemos sentado en 3.3, el futuro se formaba en el mochica añadiendo la partícula ca (ka) o choem según Carrera y chäm de acuerdo a Middendorf a fines del siglo XIX.

En los ejemplos que presentamos a continuación se observa que el significado de futuro está indicado por la presencia de la partícula ka como morfema no ligado, que funciona como un constituyente de una construcción sintáctica.

$\begin{array}{lllll}1 \mathrm{p} & \text { moin } & \mathrm{e} & \mathrm{ka} & \text { 'yo seré' } \\ 2 \mathrm{p} & \text { tsang } & \text { e } & \mathrm{ka} & \text { 'tú serás' } \\ 3 \mathrm{p} & \text { aio-f' } & & \mathrm{ka} & \text { 'él será' }\end{array}$

Dado que esta partícula se utiliza también en la formación del subjuntivo y el condicional, podemos suponer que esta partícula era una clase de adverbio o modal que significaba 'posibilidad' o 'condición'.

Tanto Carrera [1644] (1939) como Middendorf (1892) presentan el llamado "segundo tipo de futuro" formado por la adición de choem (que representaremos siguiendo a Middendorf por chäm). Esta partícula, además del significado de futuro, expresa 'deber' y 'obligación'; rasgos semánticos que pueden constituir retención del significado lexical:

$\begin{array}{lllll}1 \mathrm{p} & \text { moin } & \text { e } & \text { chäm } & \text { 'yo seré, debo ser, tengo que ser' } \\ 2 \mathrm{p} & \text { tsang } & \text { as } & \text { chäm } & \text { 'tú serás, tú debes ser' } \\ 3 \mathrm{p} & \text { aio-f } & & \text { chäm } & \text { 'él será, el deberá ser' }\end{array}$

La evolución de las formas libres a las formas ligadas parece empezar a manifestarse a fines del siglo XIX (1892), tal como sugieren los datos presentados por Middendorf:

chin-ka 'yo seré'

chis-ka

chin-ka 


\author{
chin-chäm 'yo seré; tengo que ser' \\ chis-chäm \\ ching-chäm
}

Además, tanto Carrera como Middendorf atestiguan una forma de futuro con la presencia de $\underline{\mathbf{t}}$ cuyo status en la gramática no es muy transparente. Tal vez constituya una raíz monosegmental que funciona como una raíz. Este elemento ocurre en el primer orden, al cual se añade la flexión de persona seguida por chi:

t- flexión de persona -chi

Por ahora no podemos desentrañar su significado lexical, aunque podemos especular que debe estar relacionado con el significado de deseo, posibilidad, obligación, intención, que son las posibles fuentes del significado del futuro.

\title{
5. LAS BASES LÉXICAS Y DESARROLLO DEL SIGNIFICADO DEL FUTURO
}

Sobre la base de los resultados del enfoque interlingüístico de la presente investigación, postulamos que el futuro en las lenguas de los Andes Centrales es el resultado del cambio semántico y fonético a partir de un conjunto restringido de ítems lexicales en el pre-proto-lengua. Los datos de esta investigación y las tendencias a través de las lenguas apoyan esta hipótesis (Bybee y Pagliuca, 1985b).

El significado básico del futuro al referirse a un hecho posterior al momento del enunciado por el hablante es la 'predicción'.

Al lado de la predicción aparecen otros significados secundarios como el de deseo, obligación, movimiento, intención, comando, necesidad, suposición, sugerencia. Estos significados coexisten en ciertos casos con el significado de predicción que es el significado fundamental del morfema flexivo del futuro.

En el quechua la fuente léxica es el verbo de movimiento ša- que tiene un significado básico 'movimiento' y que adquiere significados específicos con determinados sufijos derivacionales. Esta fuente está limitada a la formación del morfema flexivo de 1p y funciona combinada con el propositivo que significa 'propósito de realizar una acción'.

El marcador de la $3 p$ (-nqa) proviene de qa que se añade al verbo con marca del residuo de -na nominalizador o desiderativo $3 p(-n)$ o se asume que en la etapa del pre-protoquechua qa constituía un morfema no ligado, es decir no era un sufijo, sino un elemento de posposición.

Los ejemplos en los cuales aparecen involucrados significados resultantes de la retención, nos conducen a suponer que el sufijo de tercera persona es el desarrollo de un elemento que indicaba obligación, deseo o necesidad.

El marcador del futuro inclusivo -šun, por los significados secundarios, además del fundamental de "predicción", conduce a postular que éste es la evolución del elemento lexical que significaba obligación, comando, incitación, invitación y cortes. 
Para el aimara, en base a las evidencias presentadas, se postula que el sistema flexivo de futuro resulta de la evolución de ña que también es marca del infinitivo y del posesor ni. El primer elemento lexical era el indicador deseo, posibilidad y obligación.

En el jaqaru, además del posesor -ni de 3p, los marcadores -na(1p) y -ma (2p) han evolucionado de los marcadores de la forma del 'obligativo', que obviamente tienen el significado de obligación, significado que coexiste actualmente con el de predicción en los marcadores del futuro en esta lengua.

Respecto al mochica, los datos exhiben la condición léxica de los marcadores del futuro, constituyendo esta situación un respaldo de nuestra hipótesis y una evidencia de la hipótesis que sostiene que los estudios interlingüísticos exhiben patrones o tendencias universales en la formación del significado gramatical (Bybee y Pagliuca, 1985a), siendo el futuro el caso más patente. El caso del mochica constituye evidencia de la hipótesis de la formación del significado gramatical a partir de fuentes léxicas, por cuanto los dos elementos marcadores del futuro ka y chäm son partículas que funcionan como morfemas no ligados y que mantiene su significado más temprano (posibilidad, deseo, obligación) además del significado de futuro que se supone es el resultado del cambio del significado lexical en el significado de predicción.

Este trabajo no puede hacer ninguna predicción específica sobre la fuente léxica del futuro en el puquina por carecer de los datos necesarios. Sin embargo, la tendencia universal en la formación del futuro mostrada por los estudios interlingüísticos nos lleva a asumir que el proceso de formación del tiempo futuro en esta lengua debe haber seguido la indicada tendencia universal enunciada más arriba.

Los datos utilizados y el análisis diacrónico, así como el análisis sincrónico necesario, llevado a cabo, sugieren que los marcadores flexivos de las lenguas objeto de la presente investigación constituyen los resultados del proceso del cambio semántico a partir de un conjunto limitado de ítems lexicales cuyos significados indican deseo, intención, obligación, necesidad, suposición, comando, posesión y movimiento. El significado de futuro, es decir de predicción, es el desarrollo desde estos significados. Este proceso de cambio semántico consiste en la extensión semántica de tales significados y algunos de los significados lexicales que acompañan al significado gramatical de futuro son retenciones de los significados de las fuentes (Bybee y Pagliuca, 1985b). En el desarrollo del significado del futuro, el de la predicción llega a ser central y los significados retenidos de las fuentes son significados complementarios o secundarios.

\section{CONCLUSIONES}

Los resultados de la presente investigación han sido alcanzados mediante la comparación de las lenguas no relacionadas genéticamente, excepto entre el aimara y el jaqaru que han sido considerados para esta comparación como una sola unidad lingüística. Sin embargo estas lenguas de los Andes Centrales conforman un área lingüística y por tanto se encuentran relacionadas arealmente. 
La formación de los paradigmas flexivos de las lenguas de los Andes Centrales consiste en la evolución semántica dirigida al desarrollo del significado gramatical. Este cambio semántico consiste en la evolución de los significados lexicales en la derivación del significado del futuro, es decir de predicción. A partir de los significados lexicales de las fuentes del futuro se va desarrollando la predicción hasta convertirse en significado central. Este cambio semántico consiste en el cambio del significado lexical en significado gramatical. Este proceso del cambio semántico se denomina gramaticalización (Bybee y Pagliuca, 1985a, Sweetser, 1988, Heine et al., 1991). El cambio del significado lexical en su correspondiente gramatical implica un proceso de generalización o universalización del significado. Es decir, en la formación de los marcadores del futuro se ha producido una mutación del significado lexical, por tanto particular, al significado gramatical, denominado gramaticalización, que constituye una generalización semántica. De acuerdo a nuestra hipótesis, el proceso de la evolución semántica en el desarrollo del futuro en las lenguas estudiadas se ha cumplido de acuerdo a ciertos pasos: significado lexical $\longrightarrow$ significado gramatical $\longrightarrow$ generalización del significado.

Al lado de este proceso semántico se ha encontrado una correlación entre el grado de generalización semántica del morfema gramatical (Bybee y Pagliuca, 1985 a) y el grado de reducción y/o fusión fonética con otros ítems, en este caso con la raíz verbal.

De otro lado, debemos llamar la atención del carácter monosilábico de los morfemas del pre-proto-quechua que otros autores ya han sugerido. Este carácter explicaría que sufijos como -ša, -qa y -šun para el quechua y -ña, -na, -ni y -ma para la familia aimara -jaqaru habrían conformado morfemas no ligados y que estos habrían constituido la fuente lexical de los marcadores de futuro en las indicados lenguas. Este fenómeno reforzaría nuestra hipótesis que sustenta esta investigación.

Finalmente, debemos señalar que tanto el quechua y la familia aimara usan la persona inclusiva en oposición a la exclusiva. Este rasgo es un fenómeno tipológico determinado por la relación areal por un período prolongado. Esto nos lleva a postular que dicho rasgo puede ser independiente de la relación genética entre ambas lenguas. 
NORTE CENTRO

SUR

\begin{tabular}{|c|c|c|c|c|c|c|c|c|}
\hline & Ecuador & Chachap & Cajamarca & Sihuas & Conchucos & Huanca(Junín) & Cuzco & Ayacucho \\
\hline $1 \mathrm{p}$ & -ša & -ša & -šaq & -šaq & -sa: -šaq & -šaq & - saq & - saq \\
\hline $2 p$ & -nki & -nki & -nki & -nki & $-n k i$ & $-n k i$ & & -nki \\
\hline $3 p$ & - nqa & - nqa & -nqa & - nqa & - nqa & - nqa & $-n q a$ & - nqa \\
\hline Incl. & -nči - šun & -šun & -šun & -šun & -šun & -šun & -sun & -sun \\
\hline Excl. & & & rišaqǰapa & -ya:šaq & $\begin{array}{c}\text {-yašayki } \\
\text {-yaša: -yašaq }\end{array}$ & -paku-šaq & -saq-ku & -saq-ku \\
\hline $2 \mathrm{ppl}$ & -nkiči - & & ringī̃apa & -yanki & -yanki & -pa:kunki & -nki-čis & -nki-čik \\
\hline $3 p p l$ & -naunka & & ringajaapa & -yanqa & -yanga & -pa:ku-nqa & $-n q a-k u$ & -nqa-ku \\
\hline
\end{tabular}

\section{CUADRO 1}

Marcadores de futuro en los dialectos quechuas representativos de las tres regiones (NORTE, CENTRO Y SUR) 


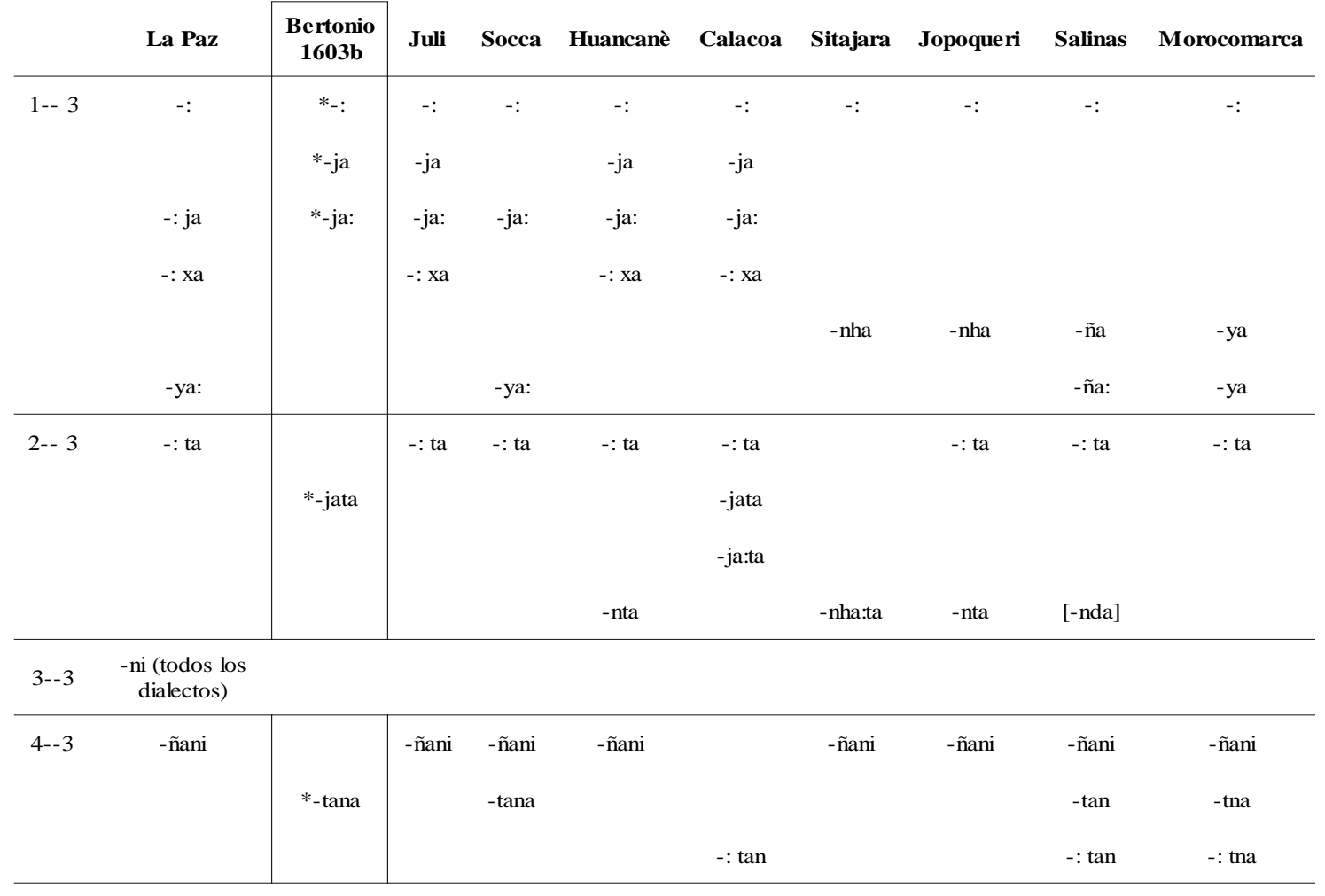

\section{CUADRO 2}

Distribución de los marcadores del futuro en los dialectos aimaras

Reproducción de la tabla 6-3 de Briggs 1976: p. 394 


\begin{tabular}{|c|c|}
\hline \multicolumn{2}{|c|}{ ELFUTURO EN EL JAQARU } \\
\hline $1 \mathrm{p}$ & -nha [-na] \\
\hline $2 \mathrm{p}$ & -mata \\
\hline $3 \mathrm{p}$ & - ni \\
\hline $4 \mathrm{p}$ & - tana (inclusivo) \\
\hline
\end{tabular}

CUADRO 3

FUTURO EN EL MOCHICA (Carrera, 1644[1939])

\begin{tabular}{lllll} 
& \multicolumn{3}{c}{ Singular } & \\
$1 \mathrm{p}$ & moin & e & ca & "yo seré" \\
$2 \mathrm{p}$ & tzhang & c & ca & "tu serás" \\
$3 \mathrm{p}$ & aiof & ca & "aquel será" \\
$1 \mathrm{pl}$ & maich ang & & & \\
$2 \mathrm{pl}$ & tzhaichez & ca & "nosotros seremos" \\
$3 \mathrm{pl}$ & aiong an & e & chica & "vosotros seréis"
\end{tabular}




\section{SEGUNDA FORMACIÓN DEL FUTURO}

\begin{tabular}{llll} 
& & \multicolumn{2}{c}{ Singular } \\
$1 \mathrm{p}$ & moin $\mathrm{c}$ & chäm & "yo tengo que ser" \\
$2 \mathrm{p}$ & tzhang az & chäm & "tu tienes que ser" \\
$3 \mathrm{p}$ & aiof & chäm & "aquel tiene que ser" \\
$1 \mathrm{pl}$ & maich eix & chäm & "nosotros tenemos que ser" \\
$2 \mathrm{pl}$ & tzhaichaz chi & chäm & "nosotros tenéis de ser." \\
$3 \mathrm{pl}$ & aiong an e & chäm & "aquellos tienen de ser"
\end{tabular}

TERCERA FORMA DEL FUTURO MIDDENDORF (1892): ANTEPOSICIÓN DE t- A LAS DESINENCIAS

$\begin{array}{llll}1 \mathrm{p} & \text { tin } & \text { met } & \text { "yo traeré" } \\ 2 \mathrm{p} & \text { taz } & \text { met } & \text { "tu traerás" } \\ 3 \mathrm{p} & \text { tang } & \text { met } & \text { "el traerá" } \\ 1 \mathrm{pl} & \text { tix } & \text { met } & \text { "nosotros traeremos" } \\ 2 \mathrm{pl} & \text { taz } & \text { metchi } & \text { "aquellos traeremos" } \\ 3 \mathrm{pl} & \text { tang } & \text { metan } & \text { "aquellos traerán" }\end{array}$

CUADRO 4 


\section{REFERENCIASBIBLIOGRÁFICAS}

BERTONIO, Ludovico. (1603[1879]). Arte de la lengua aymara. Publicada por Julio Platzman en edición facsimilar. Leipzig: B.G. Teubner.

BRIGGS, Lucy T. (1976) Dialectal Variation in the Aymara Language of Bolivia and Peru. Ann Arbor, Michigan: University Microfilms International. 2vols.

BÜTTNER, Thomas T. (1983). Las lenguas de los Andes Centrales. Madrid: Ediciones Cultura Hispánica.

BYBEE, Joan L. \& PAGLIUCA, William. (1985a). Cross-linguistics comparison and the development of grammatical meaning. In J. Fisiak (ed.)(1985). Historical Semantics, Historical Word-Formation, pp. 59-83. Berlin: Mouton de Gruyter.

(1985b). The evolution of future meaning. In A. G. Ramat, O. Carruba \& G. Bernini (eds.) (1987) Papers from the $7^{\text {th }}$ International Conference on Historical Linguistics, p. 109-122. Amsterdam: Benjamins.

CARRERA, Fernando de la (1644[1939]). Arte de la lengua yunga. Ed. Por Radames Altieri. Tucumán: publicaciones especiales del Instituto de Antropología, Universidad Nacional de Tucumán.

CERRÓN-PALOMINO, Rodolfo. (1982). El problema de la relación quechua-aru: estado actual. Lexis 6(2): 213-242.

.(1993). Quechuística y aimarística: una propuesta terminológica. Alma Mater 5: 41-55. Lima: Universidad Nacional Mayor de San Marcos.

GRASSERIE, Raoul de la. (1894). Langue Puquina. Leipzig: K.F. Koehler, Libraire-Editeur.

HARDMAN DE BAUTISTA, Martha. (1975). El jaqaru, kawki y el aymara. Actas del III Simposio del PILEI, pp. 185-192. Méjico: Editorial Galache.

.(1983). Jaqaru. Compendio de estructura fonológica y morfológica. Lima: Instituto de Estudios Peruanos e Instituto Indigenista Interamericano.

HUAYHUA PARI, Felipe. (1991). El sistema deíctico verbal del aimara. Tesis de Licenciatura en Lingüística. Lima: Facultad de Letras y Ciencias Humanas. Escuela Académico Profesional de Lingüística. UNMSM.

HEINE, Bernd, ULRIKE, Claudi \& HUNNEMEYER, Friederike. (1991). Grammaticalization. A conceptual framework. Chicago: University of Chicago Press.

MIDDENDORF, Ernst W. (1892). El mochica o la lengua chimú. In Las Lenguas Aborígenes del Perú. Tomo VI. Leipzig: F. A. Brockhaus. Traducción de Gertrud Schumacher de Peña (Manuscrito).

PADRES DE MARYKNOLL; TRACY, Emmete. \& POINT, Grosse. (1969). Lecciones de aymara. Primer nivel. Cochabamba: Instituto de Idiomas, Padres de Maryknoll.

PARKER, Gary J. (1969). Comparative Quechua Phonology and Grammar II: Proto-Quechua Phonology and Morphology. Working Papers in Linguistics, 1(2): 123-147.

.(1976). Gramática Quechua: Ancash-Huaylas. Lima: Ministerio de Educación e Instituto de Estudios Peruanos.

SWEETSER, Eve Eliot. (1988). Grammaticalization and semantic bleaching. Berkeley Linguistics Society 14: 389-405. 
QUESADA CASTILlO, Félix. (1985). Phonological Processes in Quechua and their Implications for the Phonological Theory. Buffalo, New York: State University of New York. Ph. D. Dissertation.

TORERO, Alfredo. (1970). Lingüística e historia de la sociedad andina. In A. Escobar (Comp.). El reto del multilingüismo en el Perú, pp. 51-106. Lima: Instituto de Estudios Peruanos.

Recibido: 01/07/2006

Aceito: 30/10/2006 